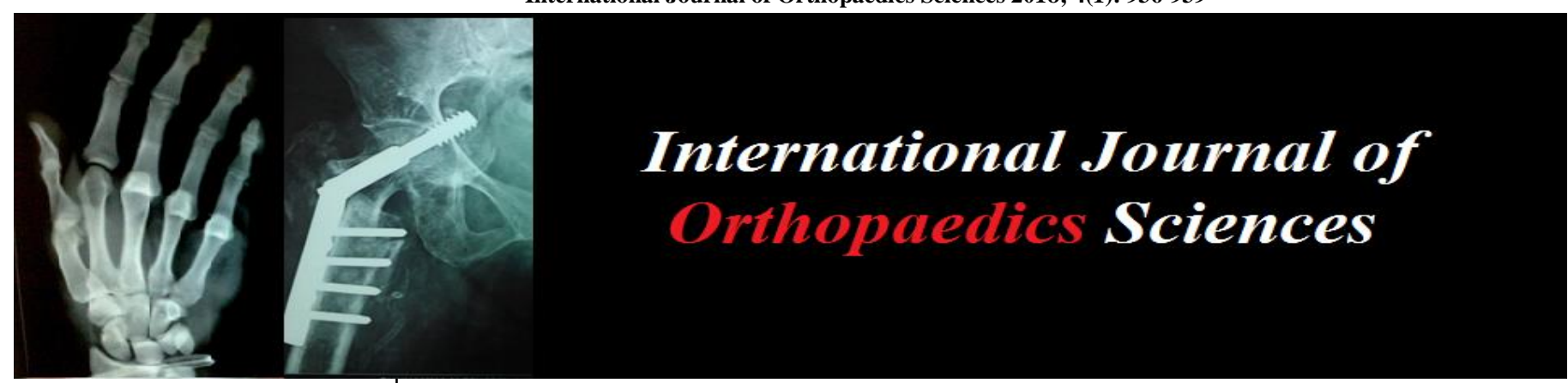

ISSN: $2395-1958$

IJOS 2018; 4(1): 936-939

(C) 2018 IJOS

www.orthopaper.com

Received: 24-11-2017

Accepted: 25-12-2017

Dr. Siddaram N Patil

Associate Professor, Sri Devraj

Medical College, Tamaka, Kolar,

Karnataka, India

Prabhuling Reddy Patil

Sri Devraj Medical College,

Tamaka, Kolar, Karnataka,

India

Dr. Ronak Daglia

Sri Devraj Medical College,

Tamaka, Kolar, Karnataka,

India

\section{A prospective study of delta frame as external fixator for open-distal tibial fractures}

\author{
Dr. Siddaram N Patil, Prabhuling Reddy Patil and Dr. Ronak Daglia
}

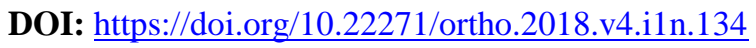

\section{Abstract}

Distal tibial fractures pose a serious challenge to the orthopaedician because muscular coverage is very less and poorly vascularized ${ }^{[1]}$. It is further complicated by comminution, joint involvement, open fractures and high energy trauma ${ }^{[1]}$. The goals of management are proper care of the injured soft tissues, restoration of joint congruity, stable fixation ${ }^{[2]}$. Ankle spanning external fixation ie Delta frame has become the initial treatment of choice for complex tibial pilon fractures ${ }^{[3]}$. The 'tripolar configuration' allows facilitation of wound care, plastic procedures and good xray or CT images ${ }^{[4]}$. The Delta external fixator was found to be the most stable external fixator for treating not stable as type III according AO/OTA's classification ${ }^{[5]}$. The IM nails often do not provide enough stability in distal tibial fractures and plating requires extensive soft tissue dissection ${ }^{[6]}$. External fixators can also be used in osteoporotic and unreconstructable comminuted metaphyseal fractures with poor bone stock ${ }^{[6]}$. However literature support is scanty, hence we decided to do the above study with an aim to analyze its results using the American Orthopaedic Foot and Ankle Society (AOFAS) Ankle Hindfoot score.

Keywords: Delta frame, external fixator, open fracture, IM nail

\section{Introduction}

Materials and Methods

The study included twenty cases of open distal tibial fractures treated by Delta frame external fixator at R. L. Jalappa Hospital attached to Sri Devaraj Urs Medical College, Kolar, and Karnataka between Jan 2015 to Dec 2017. This included a followup of six months. The inclusion criteria was skeletally mature patients with Gustilo Anderson open grade I, II, IIIA distal tibial fractures. The exclusion criteria were 1. Patients with grade IIIB and IIIC of Gustilo Anderson fractures.

2. Patients with Pelvic fractures. The data was collected from the history, clinical examination, xrays, investigations, operative notes, postoperative notes and followup notes. Complications were recorded if any. Follow up was done at 4 weeks, 8 weeks, 12 weeks, 18 weeks and 6 months. Whenever necessary patient was followed up on the telephone or called to the hospital for data collection of clinical and radiological findings. We have used the American Orthopaedic

Foot and ankle society (AOFAS) ankle hind foot scoring system.

Table 1: Showing the AOFAS scoring

\begin{tabular}{|c|c|}
\hline 1. Pain (40 points) & \\
\hline None & 40 \\
\hline Mild, occasional & 30 \\
\hline Moderate, daily & 20 \\
\hline Severe, almost always present & 0 \\
\hline
\end{tabular}




\begin{tabular}{|c|c|}
\hline \multicolumn{2}{|l|}{ 2. Function (50 points) } \\
\hline Activity limitations, support requirement & \\
\hline No limitations, no support & 10 \\
\hline $\begin{array}{l}\text { No limitation of daily activities, limitation of } \\
\text { recreational activities, no support }\end{array}$ & 7 \\
\hline Limited daily and recreational activities, cane & 4 \\
\hline $\begin{array}{l}\text { Severe limitation of daily and recreational activities, } \\
\text { walker, crutches, wheelchair, brace }\end{array}$ & 0 \\
\hline Maximum walking distance, blocks & \\
\hline Greater than 6 & 5 \\
\hline $4-6$ & 4 \\
\hline $1-3$ & 2 \\
\hline Less than 1 & 0 \\
\hline Walking surfaces & \\
\hline No difficulty on any surface & 5 \\
\hline $\begin{array}{c}\text { Some difficulty on uneven terrain, stairs, inclines, } \\
\text { ladders }\end{array}$ & 3 \\
\hline $\begin{array}{c}\text { Severe difficulty on uneven terrain, tairs, inclines, } \\
\text { ladders }\end{array}$ & 0 \\
\hline Gait abnormality & \\
\hline None, slight & 8 \\
\hline Obvious & 4 \\
\hline Marked & 0 \\
\hline Sagittal motion (flexion plus extension) & \\
\hline Normal or mild restriction $\left(30^{\circ}\right.$ or more $)$ & 8 \\
\hline Moderate restriction $\left(15^{\circ}-29^{\circ}\right)$ & 4 \\
\hline Severe restriction (less than 150 ) & 0 \\
\hline
\end{tabular}
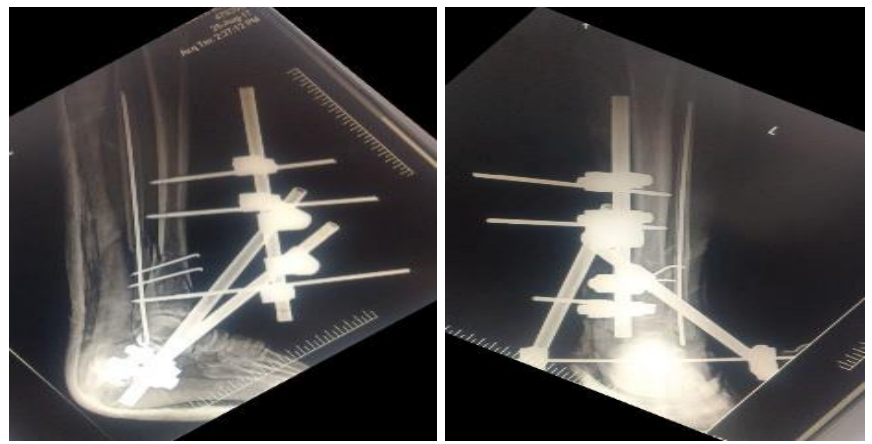

Fig 2: Immediate postop x-ray AP view Lateral View
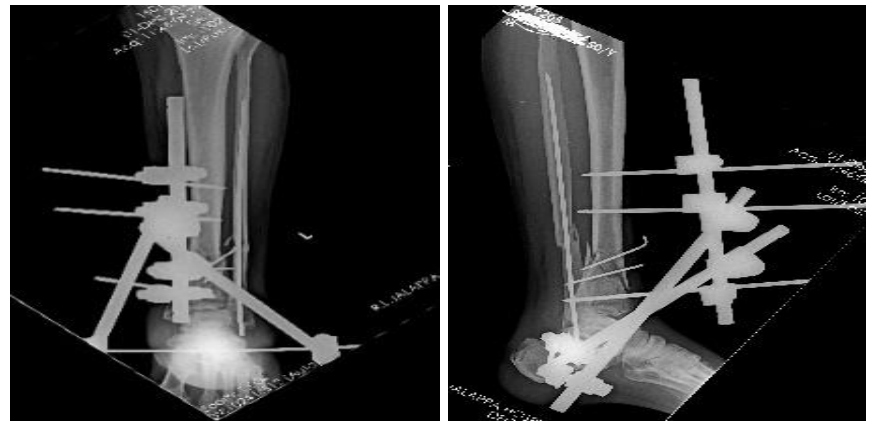

Fig 3: 5 month x-ray AP Lateral View
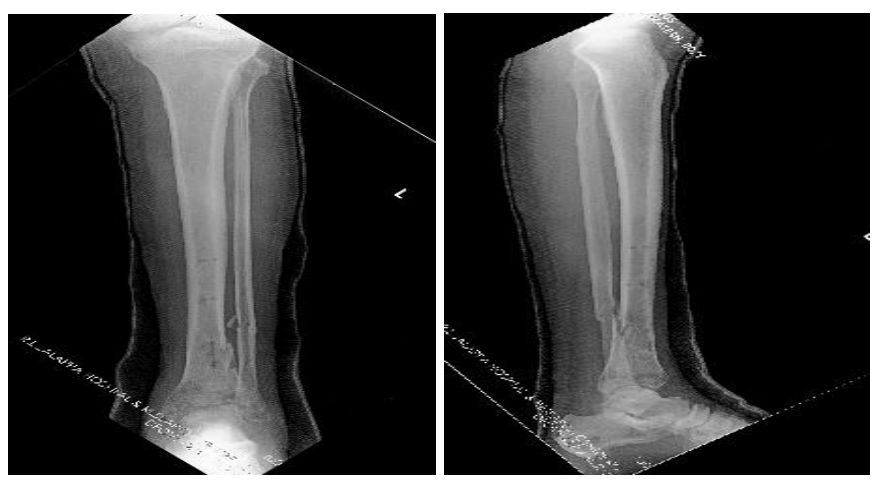

Fig 4: 6 months x-ray AP view Lat view: \# united
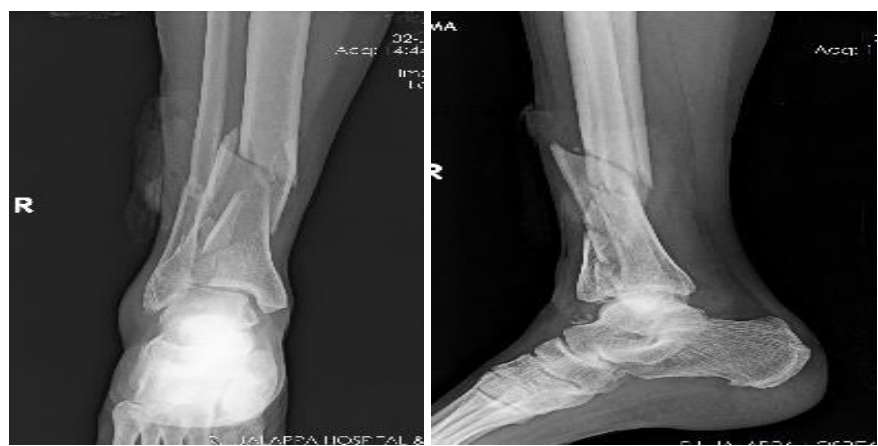

Fig 5: Preop x-ray AP view Lat view
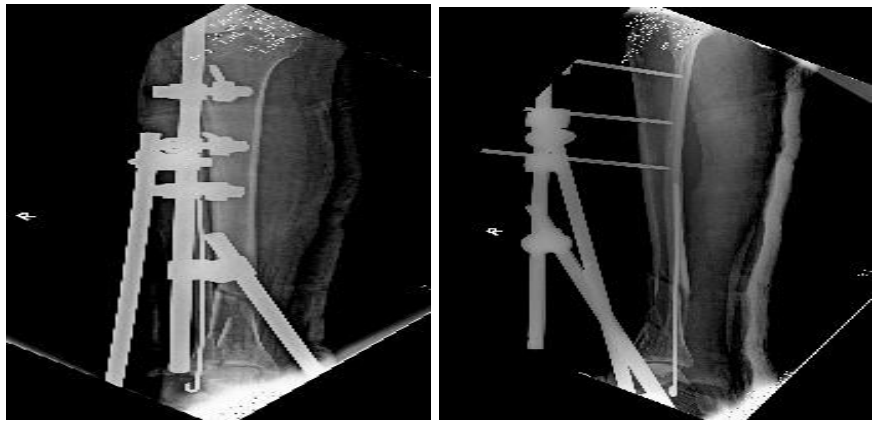

Fig 6: Immediate postop x-ray AP view Lat view
Fig 1. Preop x-ray AP 

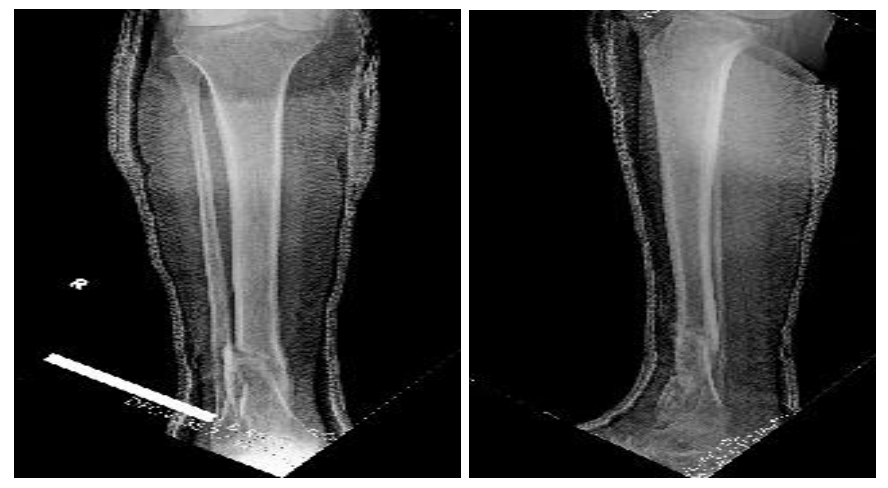

Fig 7: 5 month postop x-ray AP view Lat view and showing union
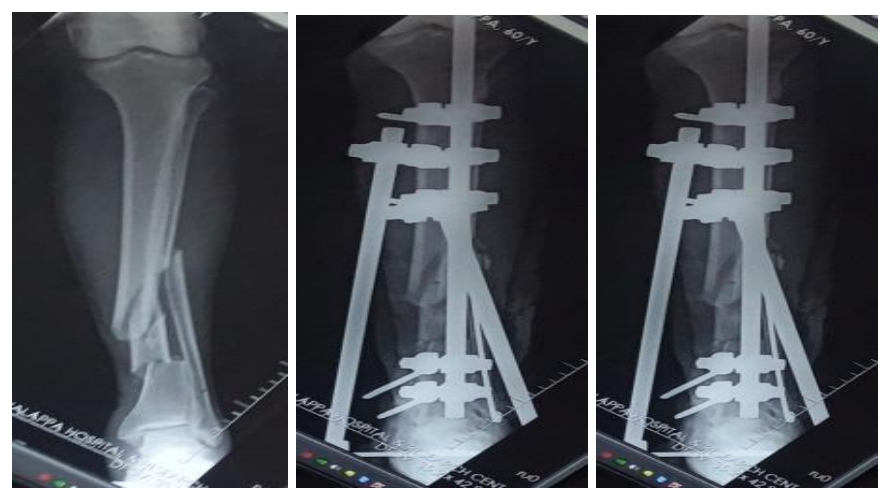

Fig 8: Preop x-ray AP view immediate postop x-ray AP view lateral view

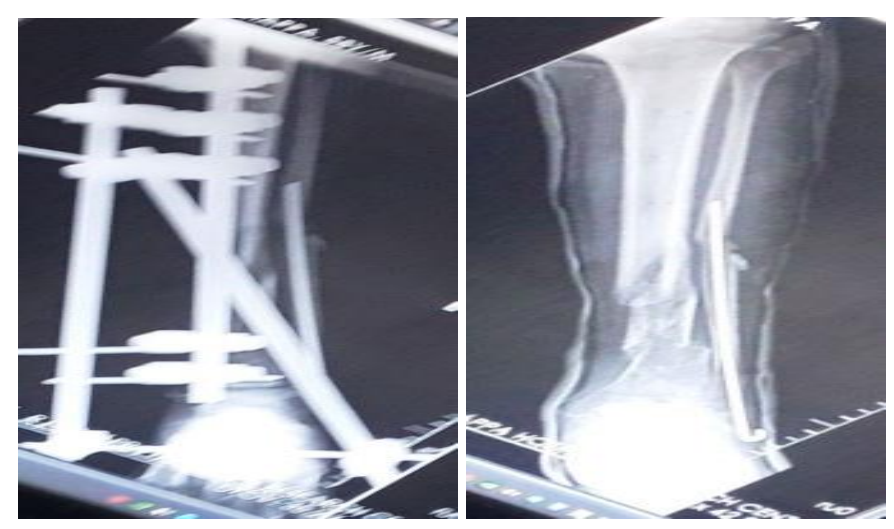

Fig 9: 4 month X-ray AP view $51 \frac{1}{2}$ month X-ray AP view

AOFAS Ankle Hind foot score was excellent in 9 good in 10 cases. Fair in 1 case, patient had Malalignment, he underwent realignment and fibular plating twelve days after index surgery Fig 9

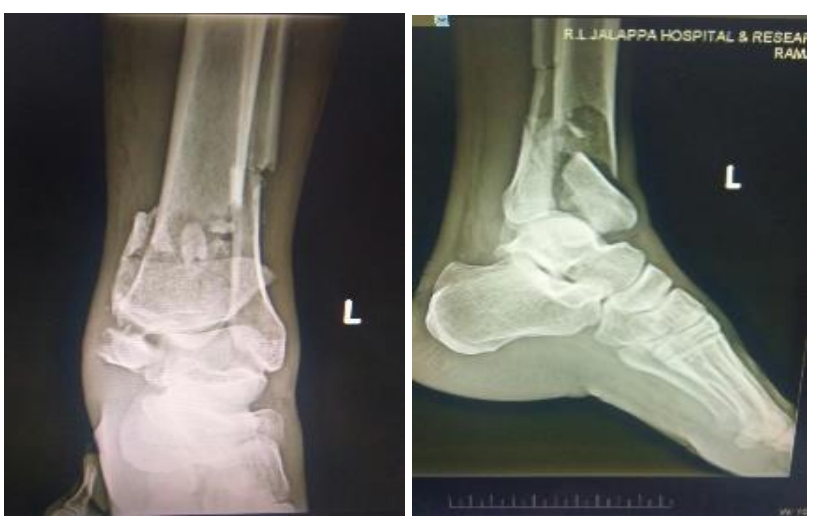

Fig 10: Preop AP view Lat view

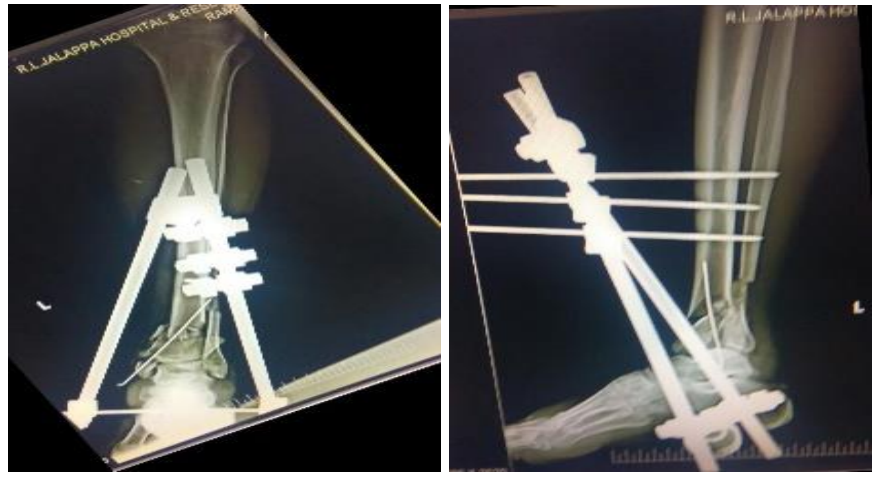

Fig 11: Immediate postop x-ray-AP view Lat view
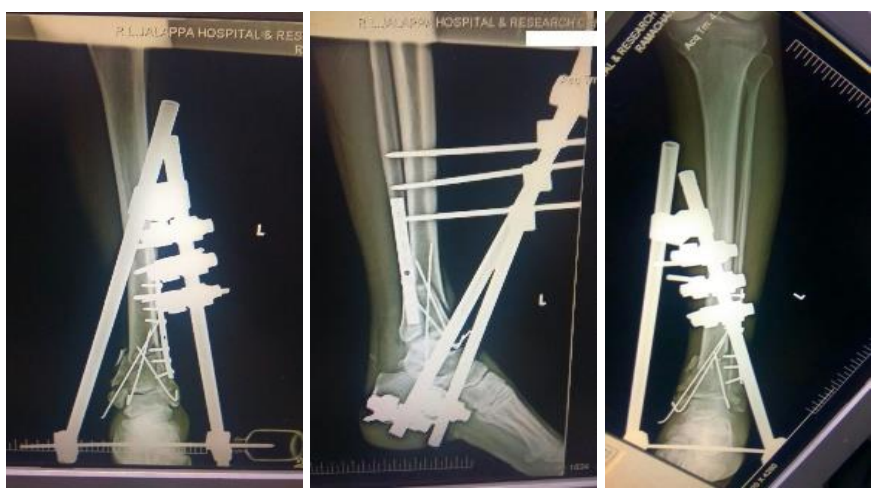

Fig 12: Plating of fibula AP view at 3 months AP view Lat view

One patient was suspected of delayed union and was converted to interlocking nailing. Fig 13

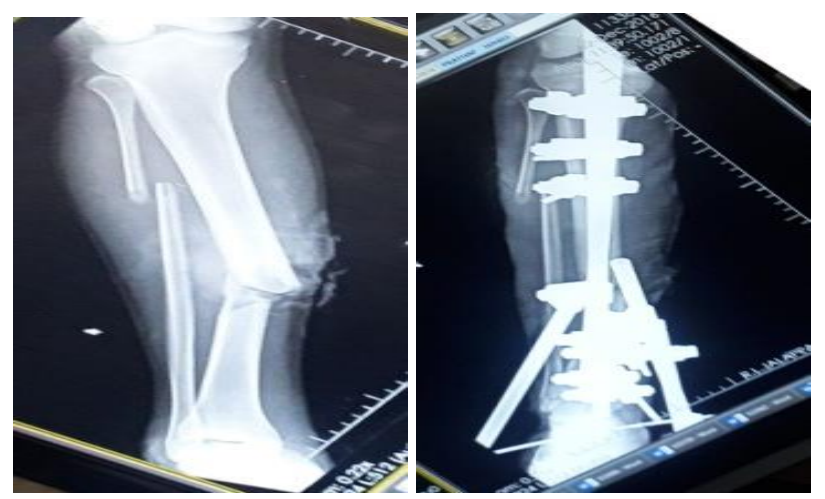

Fig x 13: Preop x-ray AP view immediate postop X-ray AP view

Fig x: Immediate postop X-ray AP view

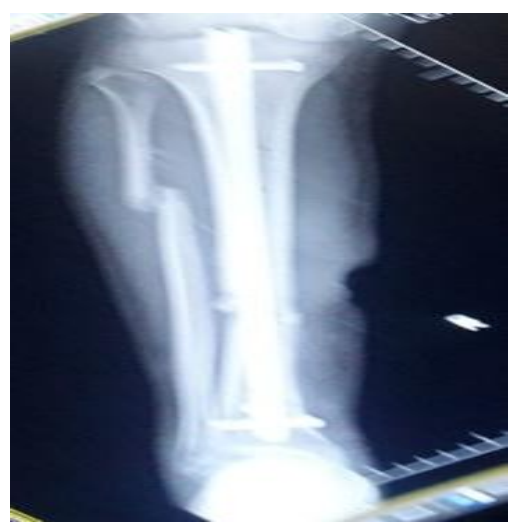

Fig 13: 3 months after nailing, showed signs of union. AP view

In another patient, we have converted into Locked compression plating. Fig 15 


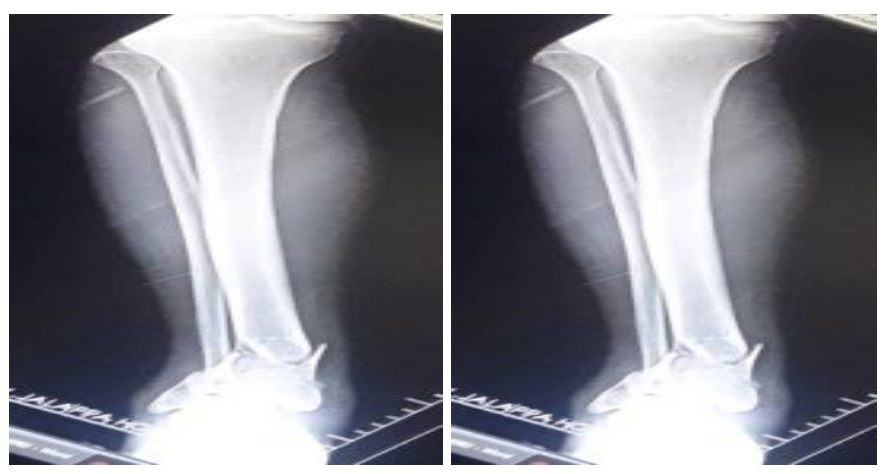

Fig 14: Preop X-ray AP view Lat view

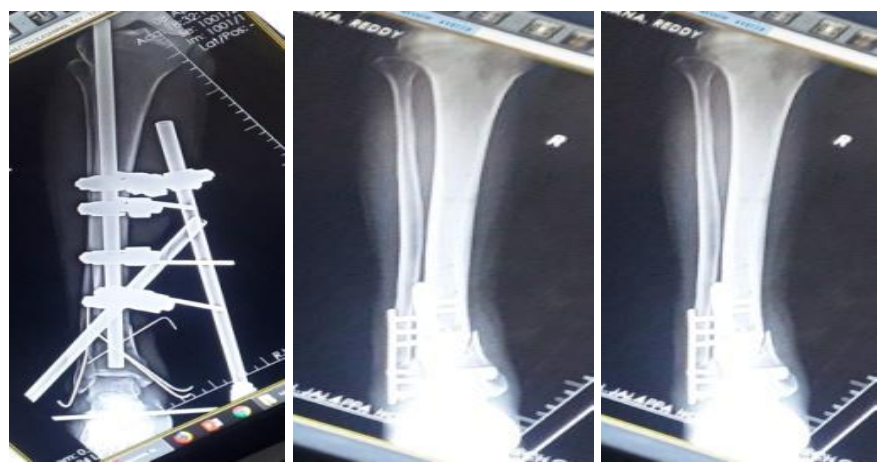

Fig 15: Immediate postop X-ray AP view Lat view 3 months after locking compression plate to tibia AP view

\section{Discussion}

Our study showed good results with Delta exfix for open distal tibial fractures. Bone et al. 1993 have used Delta frame external fixator for their twenty patients with severely comminuted pilon fractures or open pilon fractures. The time to union averaged 4.5 months, which is the same as in our study. All fractures healed. Three delayed unions required bone grafting and two had plate stabilization. Two patients required ankle arthrodesis because of posttraumatic arthritis.

In Shikari et al. study, twenty two patients with compound tibial fractures were treated with delta external fixator. Mean time to union was 5.5 months (3-16 months). AOFAS scoring showed excellent in $81 \%$. There were two delayed union and one nonunion case.

Giuseppe Rollo et al. treated 23 open tibialpilon fractures with delta frame. 13 had complications like osteoarthritis, infection and malalignment.

In the study by Venkatesh gupta et al. twenty five fractures of the distal tibia united with an average of 14.42 weeks (13-22 weeks) after hybrid external fixator. There were two cases of delayed union and no cases of non-union. 8 cases developed pin tract infections that healed with short course of appropriate antibiotics and daily antiseptic dressings. They had 12 (48\%) Cases of ankle stiffness.

Daghino et al. have performed external fixation in a 'tripolar configuration' in 36 cases. But they have used it as a temporary fixation.

\section{Conclusion}

Delta frame external fixator for open distal tibial fractures is a useful technique enabling good soft tissue healing and providing stable fixation with good results and can be used as a definitive treatment. It is especially useful when the patient has financial constraints.

\section{References}

1. Giuseppe Rollo et al. The damage control in tibialpilon open fractures with a new external Fixator delta frame. J Acute Dis. 2017; 6(5):222-6.

2. Shikari A, Wani A, Padha K, Bhatti M, Dang H. Fixation Of Compound Fractures Of Distal Tibia Using A Delta External Fixator As A Definite Modality Of Treatment With Or Without Fibular Plating/ Limited Internal Fixation With K-Wires. The Internet Journal of Orthopedic Surgery. 2010, 18(2).

3. Shikari A et al. Fixation of Compound Fractures of Distal Tibia Using a Delta External Fixator as a Definite Modality of Treatment With or Without Fibular Plating/ Limited Internal Fixation with K-Wires. Foot Ankle Int. 2007; 28(7):823-30.

4. Daghino W, Messina M, Filipponi M, Alessandro M. Temporary stabilization with external fixator in 'Tripolar' configuration in two stepstreatment of tibialpilon fractures. Open Orthop J. 2016; 10:49-55.

5. Ramlee MH, Kadir MR, Murali MR, Kamarul T. Finite element analysisof three commonly used external fixation devices for treating Type III pilon fractures. Med Eng Phys. 2014; 36(10):1322-30.

6. Venkatesh Gupta SK, Sunil G. Management of Tibial Metaphyseal Fractures by Hybrid External Fixator. Open Journal of Orthopedics. 2014; 4:84-9. http://dx.doi.org/10.4236/ojo.2014.43014 